\title{
Efficient Video Authentication for H.264/AVC
}

\author{
Jing Zhang \\ School of Electrical and Electronic Engineer \\ Nanyang Technology University \\ 639798 Singapore \\ jingzjang@pmail.ntu.edu.sg
}

\author{
Anthony T. S. Ho \\ School of Electronics and Physical Sciences \\ University of Surrey \\ Guildford, Surrey, GU2 7XH, UK \\ A.Ho@surrey.ac.uk
}

\begin{abstract}
This paper proposed a novel and efficient scheme to authenticate the H.264/AVC video. The scheme makes an accurate usage of the tree-structured motion compensation, motion estimation and Lagrangian optimization of the standard. Authentication information is embedded strictly based on the best mode decision strategy in the sense that if undergone any spatial and temporal attacks, the scheme can detect the tampering by the sensitive mode change. And the experimental results prove the effectiveness the algorithm against many transcoding and signal processing attacks.
\end{abstract}

\section{Introduction}

Digital multimedia Authentication techniques have witnessed a tremendous rise in interest over the past few years. In multimedia context, video authentication aims to establish its veracity in time, sequence and content. A video authentication system ensures the integrity of digital video, and verifies that the video taken into use has not been tampered.

In the past, several techniques and concepts based on data hiding have been introduced for tamper detection in digital images and video. One class of authentication watermarks is Hard Authentication [5]. Hard Authentication rejects any modifications to multimedia content. The in serted watermark is so weak that any manipulations to the multimedia content disturbs its integrity.

Although the concepts of hard authentication have been well studied, there are not many research works dealing with authenticating the H.264/AVC standard. With many emerging technologies, H.264/AVC has achieved a significant improvement in the enhanced compression performance, providing a factor of two in bit-rate savings when compared with existing standards such as MPEG-2 video [3]. However, whole new challenges and associated tradeoffs need to be considered for watermarking H.264/AVC data. With the exhaustive investigation of the H.264/AVC standard, we develop a novel and efficient watermarking algorithm for authentication in the compressed domain. The scheme makes an accurate usage of the tree-structured motion compensation, motion estimation and Lagrangian optimization for mode decision. The authentication information is represented by a binary watermark sequence and embedded into video frames. And the experimental results prove the effectiveness the algorithm.

The rest of this paper is organized as follows. Section 2 provides the reader with related techniques of the H.264/AVC standard. Section 3 gives a detailed explanation of the H.264 hard authentication algorithm. Some experimental results of the developed algorithm are covered in Section 4. Section 5 concludes the paper with the outline of future work.

\section{Investigation of Best Mode}

\subsection{Tree-structured Motion Compensa- tion}

Significant differences of the H.264/AVC standard from earlier standards include the support for a range of block sizes (from $16 \times 16$ down to $4 \times 4$ ) for prediction and fine sub-sample motion vectors (quarter-sample resolution in the luma component).
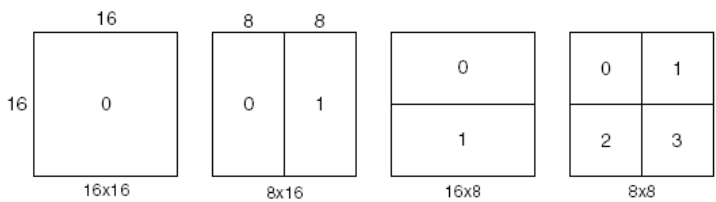

Figure 1. Macroblock partition modes: $16 \times 16$, $8 \times 16,16 \times 8,8 \times 8$ 

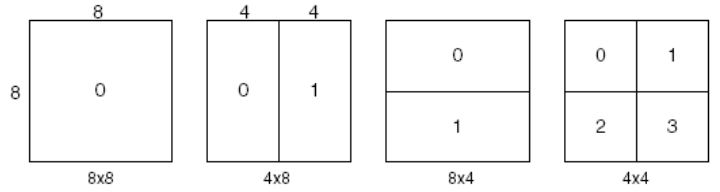

Figure 2. Sub-macroblock partition modes: $8 \times 8,4 \times 8,8 \times 4,4 \times 4$

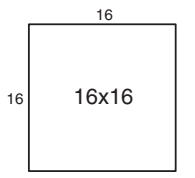

(a) mode $16 \times 16$

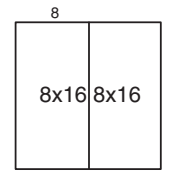

(b) mode $8 \times 16$

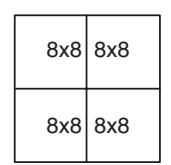

(c) mode $8 \times 8$
with 4 submodes $8 \times 8$

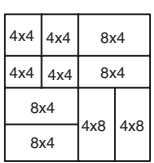

(d) mode $8 \times 8$ with submodes $4 \times 4$ $8 \times 4,8 \times 4$ \& $4 \times 8$

Figure 3. examples of partition modes using the tree-structured motion compensation

The luma component of each macroblock may be split up and motion compensated in four ways as shown in Figure 1. In cases where the $8 \times 8$ partition mode is chosen, each of the four $8 \times 8$ sub-macroblocks within the macroblock can be further split up in four ways as shown in Figure 2. This method is known as tree-structured motion compensation [4]. In general, a large partition mode is appropriate for homogeneous areas of the frame and a small partition mode may be beneficial for detailed areas. Figure 3 shows four macroblocks with different partition modes.

The best mode $I^{*}$ for a macroblock $S$ is selected by minimizing the expression in Equation (1) within the constrained $R$ and minimized $D$, using Lagrangian Optimization technique [1] of H.264/AVC, among all possible modes (denoted by $\mathcal{I}$ ):

$$
I^{*}=\arg \min _{I_{c} \in \mathcal{I}}\left(D\left(S, I_{c}\right)+\lambda R\left(S, I_{c}\right)\right)
$$

where $\lambda$ denotes the predetermined Lagrangian multiplier for mode choice, and $D$ and $R$ represent the distortion and consumed bits for encoding the current mode $I_{c}$, respectively. Figure 1 shows one frame of Foreman and the partition mode selection map.

\subsection{Partition Mode Change}

For each macroblock, the H.264 encoder selects the best partition mode among all possible partition modes that min-

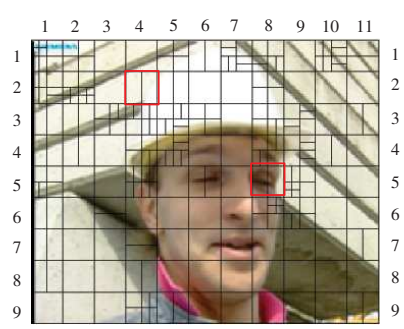

(a) mode map of the original frame

Figure 4. Foreman's partition mode selection: (a) of the original coded B-frame, (b) of the recompressed B-frame.

imizes the amount of information to be coded and sent. Figure 4. (b) shows the same frame as in Figure 4. (a) and the new partition mode selection map after recompression. From these two figures, the best partition modes of many macroblocks are not the same. For example, for the fourth macroblock in the second row, due to recompression, its best mode changes from $8 \times 16$ to $16 \times 16$. For some macroblocks, even if their best modes are the same, the information may be different. Thus the DCT coefficients may not be the same.

\section{Proposed Watermarking Method}

\subsection{Embedding}

Our algorithm is developed for the inter-predicted slices: P- and B-slices. As mentioned before, the encoder needs to find the best partition mode for each macroblock, as different modes will produce different sets of bit-rate and distortion to the video stream. The encoder will go through the motion estimation and compensation, transformation, quantization and entropy coding for all possible partition modes, and the Lagrangian optimization technique determines which partition mode has the lowest rate-distortion related cost in Equation (1). Only when the minimum cost is attained, the encoder will allocate the corresponding partition mode as the best mode to the macroblock. Through careful observation of the mode decision scheme, it can be certain that in region where there is no motion (such as background), a partition mode of $16 \times 16$ is chosen by the encoder. In areas where there is a lot of detailed motion, smaller partition modes prove to be more efficient.

Therefore, by using the mode decision scheme of the 
encoder, we could implement our watermarking algorithm targeting at higher motion activities macroblocks with the best mode $8 \times 8$ (with four sub-modes chosen from $4 \times 4$, $8 \times 4,8 \times 4$ and $8 \times 8$ ). By choosing these smaller partition modes, it is difficult for the Human Visual System to detect the differences, or the distortions introduced by the watermark embedding scheme.

For example, in one run of the iterative mode decision procedure for a macroblock $S$, if the current prediction mode $I_{c}$ is $8 \times 8$ (with four sub-modes $4 \times 4,8 \times 4,8 \times 4$ and $4 \times 8$ ) (Figure $3(\mathrm{~d})$ ), the watermark components are embedded, starting with the 10th coefficient. As the coefficients represent the residual to code, all zero coefficients are avoided to prevent the video from getting badly distorted. The nonzero quantized DCT coefficients $D(u, v)$ are replaced by the watermark components $w_{i}, i \in N$ ( $N$ is the number of nonzero coefficients):

$$
D(u, v)=w_{i}+1
$$

where $w_{i}$ is the binary authentication watermark (a pseudorandom sequence).

In the H.264/AVC standard, the encoder will first carry out the motion estimation and mode decision for modes $16 \times 16,16 \times 8$ and $8 \times 16$, for the unitary macroblock, and compute the corresponding rate-distortion related costs in Equation (1). After big partition modes, the encoder will apply the motion estimation and mode decision for mode $8 \times 8$ with the sub-modes $4 \times 4,8 \times 4,8 \times 4$ and $8 \times 8$, for four $8 \times 8$ sub-blocks. Suppose 6 watermark components are embedded, so the last watermark to be embedded is the 15 th coefficient.

After embedding watermark component $w_{i}$ into all the available modes (denoted by $\mathcal{I}$ ), the best mode $I^{*}$ for the marked macroblock $S_{m}$ is selected by minimizing the expression in Equation (1):

$$
I^{*}=\arg \min _{I_{c} \in \mathcal{I}}\left(D\left(S_{m}, I_{c}\right)+\lambda R\left(S_{m}, I_{c}\right)\right)
$$

where $\lambda, D$ and $R$ have the same meanings as in Equation (1).

After all possible modes are tested for the minimum rate-distortion cost of the current macroblock, the encoder checks whether the partition mode $8 \times 8$ with the 4 sub-mode $4 \times 4,8 \times 4,8 \times 4$ and $4 \times 8$ has the minimum cost. In case this is the best partition mode $I^{*}$, a counter of watermark sequence will be updated. And the beginning watermark component for the next macroblock will be the 16th component. However, if the rate-distortion cost is not minimal, the watermark sequence will have to redo the embedding. That is, the algorithm will have to embed the watermark components started from 10th coefficient again, when the encoder checks the rate-distortion cost of the next partition mode.

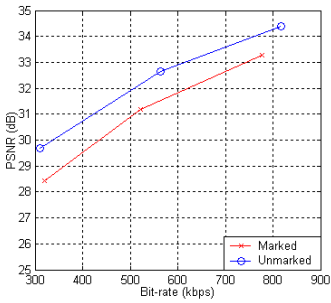

(a) Stefan

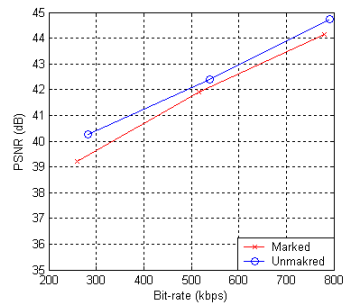

(b) News
Figure 5. Comparison of the rate-distortion performance for H.264 encoding without watermark $(\circ)$ and with watermark $(\times)$.

\subsection{Extraction}

During decoding a macroblock, only if the best mode is $8 \times 8$, all the nonzero quantized DCT coefficients (level values of entropy coding) are extracted to form the watermark sequence for authentication in a 1-D order. The extracted watermark sequence is compared to the original authentication watermark information to check if the video has been tampered.

\section{Experiments and Results}

The proposed watermarking technique has been integrated into the H.264 JM-9.0 reference software [2]. The video sequences: Foreman, Stefan, Coastguard, Mobile, Bus, Football and News are used in the experiments. All video clips are coded in CIF format $(352 \times 288$ pixels $)$ at the frame rate $30 \mathrm{frames} / \mathrm{s}$ at the bit-rate $512 \mathrm{kbit} / \mathrm{s}$. The GOP structure comprises IBPBP. . ., compliant to the Main Profile of H.264/AVC. A binary watermark sequence with 150 bits is used as the authentication information for our experiments.

Figure 5 compares the rate-distortion performance of the H.264 encoder without watermarking and with watermarking. All tests are performed with fixed quantization parameters. The unmarked and marked video clips are reconstructed from the compressed data without and with watermarks, respectively. The average PSNR values are computed by comparing the reconstructed video frames to the original raw video frames.

Two categories of video attacks have been applied to the marked video to test the sensitivity of the authentication algorithm: transcoding and common signal processing processes. 


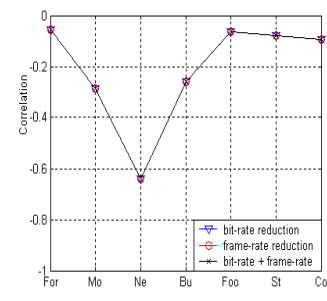

(a) Bit-rate/frame-rate/bitrate + frame-rate reduction

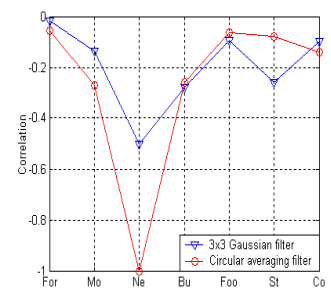

(c) $3 \times 3$ Gaussian filter and Cirucal averaging filter

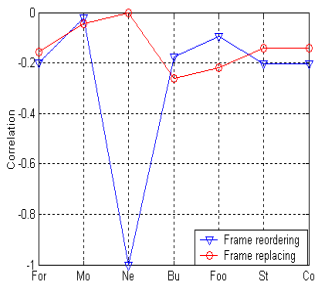

(b) Frame Reordering and replacing

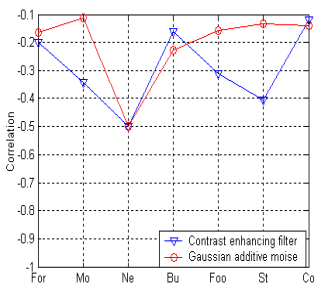

(d) Contrast enhancing filter and Gaussian Noise
Figure 6. Sensitivity under transcoding and common signal processing on Foreman, Mobile, News, Bus, Football, Stefan and Coastguard (denoted by For, $\mathrm{Mo}$, Ne, Bu, Foo, St and $\mathrm{Co}$ in the horizontal axis, respectively) (512 kbit/s, CIF-size).

In the first group, we utilized the bit-rate reduction (reducing to approximately the $1 / 2$ of the original bit-rates), frame-rate reduction (from 30 to 15 frames/s), frame reordering, and frame replacing. After decoding the marked bitstream, common signal processing attacks are applied to the raw video frame by frame, including $3 \times 3$ Gaussian low-pass filtering, circular averaging filtering, unsharpened contrast enhancement, additive Gaussian noise (mean $=0$, variance $=0.001)$.

The authentication security of our watermarking algorithm is represented by the sensitivity against attacks. The standard normalized correlation values of the original and extracted watermark sequences are measured and shown in Figure 6 , with the dynamic range from -1 to 1 . From these two figures, almost all the correlation values are less then 0 .

One special attack, the cutting and pasting attack is also applied to test the security of our authentication system. For example, the $8 \times 48$ square of $D V C$ characters in the bottom

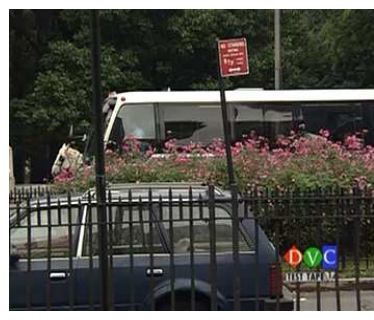

(a) Watermarked

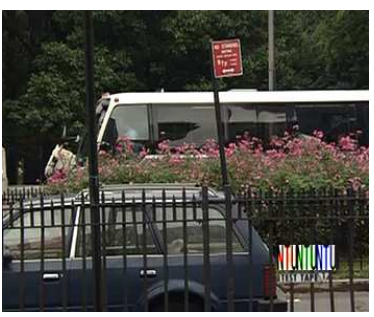

(b) undergone cutting and pasting

\section{Figure 7. The watermarked and the attacked Bus under cutting and pasting attack.}

right corner of the Bus frame is cut and replaced as shown in Figure 7: our algorithm shows the significant sensitivity at the average correlation value equal to -0.261 .

\section{Conclusions}

The proposed hard authentication algorithm performed well in terms of sensitivity against transcoding and common signal processing. The watermarked H.264/AVC video clips maintained the good visual quality and almost the same Bit-rate. However our algorithm lacks the ability to provide further information necessary to characterize the attack. Therefore, the future work will focus on enhancing the proposed algorithm by localizing the attacked areas.

\section{References}

[1] A. Joch, F. Kossentini, H. Schwarz, T. Wiegand, and G. J. Sullivan. Performance comparison of video coding standards using Lagrangian coder control. Proc. IEEE Int. Conf. Image Processing, 4:IV-3728-IV-3731, 2002.

[2] Oinline. H.264/avc joint model 9.0 (jm-9.0) reference software, available: ftp://imtc.org/jvtexports/reference_software/.

[3] T. Wiegang, H. Schwarz, A. Joch, F. Kossentini, and G. J. Sullivan. Rate-contrained coder control and compression of video coding standard. IEEE Trans. Circuits Syst. Video Technol., 13(7):688-703, 2003.

[4] T. Wiegang, G. J. Sullivan, G. Bjontegaard, and A. Luthra. Overview of the h.264/avc video coding standard. IEEE Trans. Circuits Syst. Video Technol., 13(7):560-576, 2003.

[5] B. B. Zhu, M. D. Swanson, and A. H. Tewfik. When seeing isn't believing. IEEE Signal Processing Magazine, 2004. 